crude drop-out rates from randomised controlled trials (RCTs) have their limitations as outcome measures. We have stated elsewhere (Hotopf et al, 1996a,b) that the important question of comparable tolerability and cost-effectiveness of SSRIs and tricyclics will not be resolved without a large primary-care-based RCT.

We deliberately did not subdivide reason for drop-out according to lack of efficacy or side-effects. This was because we believe drop-out from RCTs depends upon multiple factors and that the usual simple classification between side-effects and lack of efficacy is an over-simplification which may be especially subject to observer bias (Hotopf et al, 1996a).

We have addressed some of Lynch \& Curran's points in another paper (Hotopf et $a l, 1997$, in press), in which we assessed methodology of the RCTs. Dosages of tricyclics are often inadequately reported in trials: while some studies allow as wide a range of dosages of tricyclics as $75-300 \mathrm{mg}$ in their protocols, approximately one-quarter did not report a mean or median dose attained. Similarly, while the point at which drop-out takes place is important, it is also frequently not reported. Lynch \& Curran also mention the duration of studies as another source of heterogeneity. We believe this is less important since two-thirds of studies were of six weeks' duration.

Anderson \& Mortimore flatter us by suggesting our paper may be sufficiently widely read to bring evidence-based medicine into disrepute. Their comments mainly concern our decision to classify RCTs according to the 'generation' of tricyclic used. Evidence-based medicine aims to apply research findings to practical clinical problems. The tendency of most trials to use the oldest TCAs is of limited clinical relevance (at least in primary care) as these are not very widely used. It is more relevant to compare current commonly prescribed alternatives, and this was our rationale for the subdivision. Before new (and relatively expensive) drugs such as the SSRIs are widely prescribed, a clear advantage must be demonstrated over the older medications. The absence of any evidence that the newer TCAs have lower drop-out rates than SSRIs is, therefore, important. It is possible, perhaps even likely, that the newer TCAs do have higher drop-out rates. We mentioned the lack of power and gave confidence intervals so readers could make up their own minds, so Anderson \& Mortimore's comments regarding "statistical sophistry or naïvety" are unfounded.

Anderson \& Mortimore's third comment appears to contradict their other points. The analysis according to type of antidepressant shows that significant heterogeneity remained for the old tricyclics, but not for the other two groups, suggesting that while other sources of heterogeneity exist in the older tricyclics, they do not for the other drugs. This seems to support our classification rather than to refute it. There will always be uncertainty regarding the investigation of heterogeneity (Thompson, 1994) and there may be more than one feasible explanation for any given meta-analysis.

Hotopf, M. H., Lewis, G. \& Normand, C. (1996a) The Treatment of Depression: Evaluation and Cost-Effectiveness. London: London School of Hygiene and Tropical Medicine.

_. _ _ (1996b) Are SSRIs a cost-effective alternative to tricyclics? British journal of Psychiatry, 168, 404-409.

_ , _ _ (1997) Putting trials on trial: the costs and consequences of small trials in depression: a systematic review of methodology. Journal of Epidemiology and Community Health, in press.

Thompson, S. G. (1994) Why sources of heterogeneity in meta-analysis should be investigated. British Medical journol. 309. $1351-1355$

M. Hotopf, R. Hardy, G. Lewis King's College School of Medicine \& Dentistry and the Institute of Psychiatry, Department of Psychological Medicine, 103 Denmark Hill, London SE5 8AZ

\section{Cost-effectiveness of antidepressant treatment}

Sir: We read with interest Woods \& Rizzo's (1997) re-examination of Jönsson \& Bebbington's (1994) model for comparing the cost-effectiveness of antidepressants. They concluded that tricyclic antidepressants (TCAs) are more cost-effective than selective serotonin reuptake inhibitors (SSRIs) as initial treatment for depression. Neither of these papers, nor the recent review in this journal by Hotopf et al (1996), included the costs associated with antidepressant overdose in their comparisons. We believe that while some attention has been paid to the mortality associated with TCA overdose there has been little consideration of its morbidity and the consequent costs.

In an audit of 404 cases of deliberate self-harm presenting to the accident and emergency department at Addenbrooke's Hospital, Cambridge, in the six-month period between 1 January and 30 June
1996 there were 75 cases of deliberate selfpoisoning with antidepressants. There were 35 cases of deliberate self-poisoning with TCAs and $\mathbf{4 0}$ cases of SSRI overdose. In one case the antidepressant was not identified, one case was of deliberate self-poisoning with trazodone and in two cases an SSRI was taken with a TCA. Thirty of the 35 cases of TCA overdose were admitted to Addenbrooke's Hospital, including six who were admitted to the intensive therapy unit (ITU) and three admitted to the cardiac monitoring unit (CMU). One of these patients died in ITU. Of the subgroup of 16 cases involving newer TCAs (lofepramine and dothiepin), 13 were admitted to Addenbrooke's including two admitted to ITU and one admitted to CMU. Twenty-five of the 40 cases of SSRI overdose were admitted to Addenbrooke's and none of these was admitted to ITU or CMU.

The Addenbrooke's Hospital NHS Trust has recently costed a bed in ITU for one day at $£ 1530$ and a bed in CMU at $£ 340$. These figures represent costs approximately 15 times and three times, respectively, those of a bed on a general ward. This audit indicates that the cost of treating antidepressant overdoses is considerably greater for TCAs than for SSRIs.

Hotopf, M., Lewis, G. \& Normand, C. (1996) Are SSRIs a cost-effective alternative to tricyclics? British Journal of Psychiatry, 168, 404-409.

Jönsson, B. Bebbington, P. E. (1994) What price depression? The cost of depression and cost-effectiveness of pharmacological treatment. British Journal of Psychiatry, 164, 665-673.

Woods, S.W. 2 Rizzo, J. A. (1997) Cost-effectiveness of antidepressant treatment reassessed. British journal of Psychiatry, 170, 257-263.

L. Head, V. O'Keane S3 Psychiatry Outpatients, Box 175, Addenbrooke's Hospital, Hills Road. Cambridge CB2 2QQ

\section{Cognitive function and fall-related fractures}

Sir: Jabourian et al (1994) found a high prevalence of cognitive dysfunction in 120 patients admitted to a hospital exclusively because of fall-related fractures; only $12 \%$ of their sample had a normal score on the Mini-Mental State Examination (MMSE; Folstein et al, 1975). Based on these findings, Jabourian et al argued that cognitive impairments are a major risk factor for falls. It is questionable whether all patients with 